\title{
LAS ESCALAS DE DEPRESIÓN, ANSIEDAD Y ESTRÉS (DASS-21): ¿MIDEN ALGO MÁS QUE UN FACTOR GENERAL?
}

\section{The Depression Anxiety Stress Scales (DASS-21): do they measure anything beyond a general factor?}

\author{
Pablo D. Valencia* \\ Asociación Peruana Contextual - Conductual de Psicología
}

\begin{abstract}
Resumen
El solapamiento entre depresión y ansiedad es un hallazgo ampliamente replicado en la investigación sobre salud mental. Las Escalas de Depresión, Ansiedad y Estrés (DASS-21) fueron desarrolladas para maximizar la discriminación entre estos constructos. Sin embargo, la investigación sugiere que las DASS-21 miden predominantemente un factor general de malestar emocional. En este estudio, se tradujo las DASS-21 y se aplicaron a 353 universitarios (Medad = 20.42; 61 \% mujeres) de Lima, Perú. El análisis factorial confirmatorio apoyó un modelo bifactor, pero análisis adicionales mostraron que los datos eran esencialmente unidimensionales. Más aún, cuando los ítems fueron sometidos a un análisis exploratorio bifactor, algunos no cargaron en sus factores específicos originales. Se concluye que las DASS-21 deben ser interpretadas como una dimensión global de malestar emocional.
\end{abstract}

Palabras clave: depresión, ansiedad, DASS-21, análisis factorial, modelo bifactor

\begin{abstract}
The overlap between depression and anxiety is a widely-replicated finding in mental health research. The Depression, Anxiety, Stress Scales (DASS-21) were developed to maximize the discrimination between these constructs. However, research suggests that DASS-21 predominantly measure an overall factor of emotional distress. In this study, DASS-21 were translated and applied to 353 university students (Mage $=20.42 ; 61 \%$ women) from Lima, Peru. The confirmatory factorial analysis supported a bifactor model, but additional analyses revealed that the data were essentially one-dimensional. Moreover, when the items were subjected to a bifactor exploratory analysis, some did not load into their original specific factors. The conclusion was that DASS-21 must be interpreted as a global dimension of emotional distress.
\end{abstract}

Keywords: depression, anxiety, DASS-21, factor analysis, bifactor model.

*Psicólogo. pabvalenciam@gmail.com.

ORCID: http://orcid.org/0000-0002-6809-1805 


\section{INTRODUCCIÓN}

Existe un elevado solapamiento entre ansiedad y depresión, el cual se observa tanto en la comorbilidad de diagnósticos clínicos como en las altas correlaciones entre medidas dimensionales de ambas variables (Barlow \& Durand, 2015; Sandín \& Chorot, 2009). Este fenómeno ocurre a pesar de que ambos términos presentan límites conceptuales claros en su definición. La depresión es definida por la presencia de un estado de ánimo de tristeza acompañado de otras alteraciones (p. ej., del sueño o del apetito), el cual ocurre de forma tal que causa malestar a quien lo padece y afecta su capacidad funcional (Asociación Americana de Psiquiatría, 2014; Vásquez \& Sanz, 2009). Por otra parte, la ansiedad se caracteriza, principalmente, por la presencia de la emoción de miedo y comprende un triple sistema de respuesta: un componente subjetivocognitivo, uno fisiológico-somático y uno motorconductual (Sandín \& Chorot, 2009).

Diversos autores han señalado la necesidad de identificar los procesos comunes y distintivos de la depresión y la ansiedad (Eysenck \& Fajkowska, 2018). Una de las propuestas que ha generado mayor investigación es el llamado modelo tripartito (Clark \& Watson, 1991). De acuerdo con este modelo, tanto la depresión como la ansiedad comparten una dimensión común denominada afecto negativo, la cual describe una tendencia global a experimental malestar (distress) emocional (Watson \& Clark, 1984). Además de esta dimensión compartida, la depresión y la ansiedad poseen, cada una, una dimensión específica. En el caso de la depresión, se observa la presencia de bajo afecto positivo o anhedonia, es decir, un interés disminuido por el entorno y una falta de activación conductual (Clark \& Watson, 1991). En la ansiedad, por otra parte, el carácter distintivo es dado por una dimensión de hiperactivación fisiológica, la cual consiste en un aumento de la reactividad autonómica y se observa en su forma más evidente en el caso del ataque de pánico (Brown, Chorpita, Korotitsch, \& Barlow, 1997).

En vista del solapamiento existente entre la depresión y la ansiedad, se han creado instrumentos que tienen por finalidad incrementar la distinción entre ambas. Un ejemplo de esto son las Escalas de Depresión, Ansiedad y Estrés (DASS), las cuales fueron construidas empíricamente - es decir, sin una concepción teórica previa - a través de la técnica del análisis factorial (Lovibond \& Lovibond, 1995). Aunque el objetivo inicial fue crear una medida de depresión y ansiedad, duranteel proceso de construcción se identificó un tercer factor, que los autores denominaron estrés, el cual describe respuestas de tensión e irritabilidad. Las DASS originales - creadas originalmente en Australia - consistían en 42 ítems (14 ítems por cada escala) y fueron luego reducidas para formar una versión breve de 21 ítems: las DASS21 (Antony, Bieling, Cox, Enns, \& Swinson, 1998). Las DASS-21 han sido traducidas a distintos idiomas: chino (Wang et al., 2016), coreano (Jun, Johnston, Kim, \& O'Leary, 2018; Lee et al., 2019), griego (Kyriazos, Stalikas, Prassa, \& Yotsidi, 2018), italiano (Bottesi et al., 2015), portugués (Patias, Machado, Bandeira, \& Dell'Aglio, 2016), ruso (Scholten, Velten, Bieda, Zhang, \& Margraf, 2017), sueco (Alfonsson, Wallin, \& Maathz, 2017), turco (Yıldırım, Boysan, \& Kefeli, 2018), vietnamita (Tran, Tran, \& Fisher, 2013), entre otros, incluido el español (Bados, Solanas, \& Andrés, 2005; Daza, Novy, Stanley, \& Averill, 2002; Gurrola, Balcázar, Bonilla, \& Virseda, 2006; Román, Vinet, \& Alarcón, 2014).

A pesar de que la estructura original de las DASS21 consta de tres dimensiones (Lovibond \& Lovibond, 1995), otros estudios han sugerido soluciones alternativas. Por ejemplo, Duffy, Cunningham y Moore (2005) hallaron que, luego de realizar algunas modificaciones al instrumento, un modelo de dos factores -activación fisiológica y negatividad general - presentaba un ajuste aceptable. Por su lado, Szabó y Lovibond (2006) hallaron que las dimensiones de ansiedad y estrés no se distinguían en niños de entre 7 y 14 años, por lo que propusieron un modelo de dos dimensiones para las DASS-21: depresión y ansiedadestrés.

Por otra parte, existe evidencia que apunta a la preponderancia de un factor general en las DASS (tanto en su versión de 42 ítems como en la de 21). Por ejemplo, la gran mayoría de estudios reporta correlaciones bastante elevadas entre los tres factores (Kyriazos et al., 2018; Le et al., 2017; Lee et al., 2019; Moussa, Lovibond, Laube, \& Megahead, 2017; Page, Hooke, \& Morrison, 2007; Patias et al., 2016; Shaw, Campbell, Runions, \& Zubrick, 2016; Sinclair et al., 
2012; Szabó, 2010; Wang et al., 2016; Willemsen, Markey, Declercq, \& Vanheule, 2011). Las elevadas correlaciones interfactoriales observadas en la mayoría de los estudios llevan a considerar la posibilidad de un factor general. Más aún, al examinar la dimensionalidad de la DASS-21 a través del análisis de componentes principales, algunos autores encontraron apoyo para la extracción de un único componente (Patrick, Dyck, \& Bramston, 2010; Sinclair et al., 2012; Tran et al., 2013). Sin embargo, cuando esta unidimensionalidad se puso a prueba con un análisis factorial confirmatorio (AFC), el ajuste fue defectuoso y solo mejoró cuando se modelaron los tres factores del modelo original, aun cuando las correlaciones entre ellos fueron muy altas (Sinclair et al., 2012). Asimismo, Camacho, Cordero y Perkins (2016) encontraron que el análisis paralelo indicaba la extracción de un solo factor al realizar un análisis factorial exploratorio. Desde otra perspectiva, algunos estudios hallaron que las tres escalas DASS-21 logran discriminar entre grupos clínicos y no clínicos, pero no entre grupos diagnósticos de ansiedad y depresión (Tran et al., 2013; Yıldırım et al., 2018). En conjunto, estos resultados sugieren la presencia de un factor general junto con algunos factores específicos de naturaleza residual, lo cual corresponde a un modelo bifactor (Reise, 2012; Reise, Bonifay, \& Haviland, 2013; Rodriguez, Reise, \& Haviland, 2016a, 2016b).

Algunos modelos bifactor probados con las DASS21 siguieron una forma no estándar (por ejemplo, permitieron errores correlacionados o correlaciones entre factores específicos), lo cual dificulta en parte su interpretación (Henry \& Crawford, 2005; Le et al., 2017; Randall et al., 2017; Szabó, 2010; Tully, Zajac, \& Venning 2009; Willemsen et al., 2011). Sin embargo, en otros estudios, se ha realizado análisis más detallados del modelo bifactor, los cuales han determinado que la mayor parte de la varianza de los ítems es explicada por un factor general (Bottesi et al., 2015; Kia-Keating et al., 2018; Moore, Dowdy, \& Furlong, 2017; Osman et al., 2012; Shaw et al., 2016). Por otro lado, algunos autores han preferido poner a prueba un modelo jerárquico, el cual consiste en un factor de segundo orden que explica las correlaciones entre los tres factores de primer orden, y al cual aplicaron una transformación Schmid-Leiman (Ruiz, García Martín, Suárez Falcón, \& Odriozola González, 2017). Sin embargo, los modelos de segundo orden se encuentran anidados en los modelos bifactor y, bajo ciertas condiciones, pueden ser equivalentes a estos cuando se aplica una transformación Schmid-Leiman (Brown, 2015). Por este motivo, no se analizó un modelo de segundo orden en el presente estudio.

El objetivo de este trabajo fue examinar, a través del análisis factorial, la dimensionalidad de la DASS-21. Para ello, se pusieron a prueba distintos modelos derivados de la bibliografía previa. La Tabla 1 describe los cinco modelos alternativos que se pusieron a prueba. El primero (M1) es un modelo unidimensional, el cual se basa en el hallazgo de que las DASS-21 son explicadas mejor por un factor preponderante de malestar emocional. El segundo (M2) se basa en el modelo de dos factores propuesto por Duffy et al. (2005). El tercer modelo (M3) agrupa los factores de ansiedad y estrés en un solo factor, lo que resulta, también, en una estructura de dos factores (Szabó \& Lovibond, 2006). El cuarto modelo (M4) es el propuesto originalmente por Lovibond y Lovibond (1995). Finalmente, el quinto modelo probado (M5) corresponde al modelo bifactor.

Tabla 1

Modelos analizados con el AFC

\begin{tabular}{lll}
\hline Modelo & \multicolumn{1}{c}{ Factores } & \multicolumn{1}{c}{ Ítems } \\
\hline M1 & 1: malestar emocional & ME: $1-21$ \\
M2 & 2: activación fisiológica & AF: $2,4,7,19$ \\
& y negatividad general & NG: $1,3,5,6,8-18,20,21$ \\
M3 & 2: depresión y ansiedad & D: $3,5,10,13,16,17,21$ \\
& -estrés & AE: $1,2,4,6-9,11,12,14,15,18-20$ \\
M4 & 3: depresión, ansiedad & D: $3,5,10,13,16,17,21$ \\
& y estrés & A: $2,4,7,9,15,19,20$ \\
& & E: $1,6,8,11,12,14,18$ \\
M5 & 4: malestar emocional, & ME: $1-21$ \\
& depresión, ansiedad & D: $3,5,10,13,16,17,21$ \\
& y estrés & A: $2,4,7,9,15,19,20$ \\
& & E: $1,6,8,11,12,14,18$ \\
\hline
\end{tabular}

\section{MÉTODO}

\section{Participantes}

Se accedió a una muestra inicial de 376 estudiantes de una universidad pública de Lima, cuyas edades se hallaron entre los 15 y los 30 años. Con el fin de reducir la heterogeneidad etaria de los participantes, se decidió recortar esta muestra inicial para incluir solo el 
rango de edad de 18 a 25 años $(\mathrm{M}=20.42, \mathrm{DE}=1.59)$. La muestra final estuvo compuesta por 353 estudiantes de Psicología (215 mujeres, $61 \%$ ) de distintos años de estudio: $32 \%$ del primer año, $27 \%$ del segundo, $24 \%$ del tercero y $18 \%$ del cuarto. Sesenta y cinco participantes (18\%) manifestaron que, al momento de la evaluación, trabajaban además de estudiar.

\section{Instrumento}

Escalas de Depresión, Ansiedad y Estrés (DASS21; Lovibond \& Lovibond, 1995). Estas forman parte de un solo cuestionario, el cual incluye siete ítems por escala. Se presenta al evaluado una serie de posibles situaciones de malestar emocional y se le pide señalar con qué frecuencia experimentó cada una de ellas durante la última semana. El instrumento brinda cuatro opciones de respuesta en formato Likert, ordenadas del 0 (No describe nada de lo que me pasó o sentí en la semana) al 3 (Sí, esto me pasó mucho, o casi siempre).

Existen diversas versiones en español de las escalas DASS-21 (Bados et al., 2005; Daza et al., 2002; Gurrola et al., 2006; Román et al., 2014). Sin embargo, al formular el presente trabajo, se observó que ninguna de ellas reflejaba fielmente los ítems de la escala original. Por ejemplo, se observó que la versión de Daza et al. (2002) omitía o reformulaba las aclaraciones que aparecen entre paréntesis en los ítems 4 y 7. Asimismo, la versión de Gurrola et al. (2006) presentaba traducciones en exceso literales, lo cual dificultaba su comprensión. A partir de estos inconvenientes con las versiones previas, el autor del presente estudio elaboró una nueva versión castellana del instrumento tomando como referencia las versiones de Román et al. (2014), Daza et al. (2002) y Gurrola et al. (2006). Esta nueva versión fue traducida al inglés, de manera independiente, por dos personas bilingües. Las dos traducciones resultantes fueron comparadas entre sí y con los ítems originales en inglés, lo cual permitió demostrar que la nueva versión castellana mantenía el sentido de los ítems originales. La comprensión de esta versión fue evaluada cualitativamente a través de aplicaciones piloto a tres grupos de personas: estudiantes universitarios, personas con diagnóstico de esquizofrenia en estabilidad clínica y personas hospitalizadas por farmacodependencia. En todos los casos, los ítems fueron comprendidos sin dificultad, por lo que no se realizó modificaciones a esta versión.

\section{Procedimiento}

La evaluación se realizó en los salones de clase de los participantes, previo acuerdo con el docente de aula y previa explicación a los participantes acerca de la naturaleza del estudio. En todos los casos, la participación en el estudio fue voluntaria y se dejó clara la posibilidad de no responder al cuestionario. Los participantes brindaron su consentimiento de manera oral. Cabe mencionar que, debido al diseño de una investigación independiente a esta, se les pidió brindar sus códigos de estudiante. Sin embargo, no se les pidió colocar su nombre y, en todo momento, se hizo énfasis en la confidencialidad de los datos.

\section{Análisis de datos}

Inicialmente, se analizaron la media y la desviación estándar de cada uno de los ítems. Sin embargo, debido a la naturaleza ordinal de estos, se examinaron, también, los porcentajes de respuesta a cada una de las cuatro categorías que presenta la prueba.

A continuación, se realizó un análisis factorial confirmatorio (AFC) utilizando el estimador de mínimos cuadrados ponderados ajustados a la media y la varianza (WLSMV), pues este procedimiento es más adecuado cuando se analizan ítems ordinales como las escalas Likert (Brown, 2015). Los cinco modelos descritos en la introducción del presente trabajo se evaluaron a través de una versión robusta del estadístico $\chi^{2}$ (WLSMV $\chi^{2}$ ), la cual, de resultar significativa, indicaría que el modelo propuesto muestra un ajuste imperfecto a los datos. Sin embargo, este estadístico puede resultar en exceso conservador, sobre todo en cuanto aumenta el tamaño de la muestra (Byrne, 2012).Por este motivo, se consideraron además los siguientes índices (se incluyen entre paréntesis los criterios que indican un buen ajuste): el índice de ajuste comparativo (CFI > .95), el índice de TuckerLewis (TLI > .95), el índice de aproximación de la raíz de cuadrados medios del error (RMSEA < .06), la raíz estandarizada del cuadrado medio del residuo (SRMR < .08) y la raíz ponderada del cuadrado medio del residuo (WRMR < 1.00; DiStefano, Liu, Jiang, \& Shi, 2018).

En relación al modelo bifactor (M5), es importante considerar si los tres factores específicos (depresión, ansiedad y estrés) poseen suficiente importancia como 
para justificar (a) la sumatoria de sus ítems como una dimensión independiente del factor general y (b) su inclusión en modelos estructurales (Reise et al., 2013). Para este fin, se ha desarrollado un conjunto de estadísticos que permiten evaluar la importancia de los factores (general y específicos) obtenidos (Rodriguez et al., 2016b). El primero de estos índices es el coeficiente omega $(\omega)$, el cual brinda información sobre la medida en que la varianza total de los puntajes compuestos puede ser atribuida a todas las fuentes de varianza común (es decir, todos los factores) del modelo. Por otra parte, el coeficiente omega jerárquico $(\omega \mathrm{H})$ indica la proporción de la varianza total que es explicada por una única variable latente (factor) del modelo. Ambos coeficientes $-\omega$ y $\omega \mathrm{H}-$ pueden ser calculados tanto para la escala general como para las subescalas.

Otro aspecto importante es determinar la medida en que un conjunto de ítems representa a una determinada variable latente. Esto se puede realizar con el coeficiente $\mathrm{H}$ propuesto por Hancock y Müeller (2001; Dominguez-Lara, 2016), el cual - al igual que $\omega$ y $\omega \mathrm{H}$ - puede calcularse para el factor general y para los factores específicos (Rodriguez et al., 2016a, 2016b). Rodriguez et al. (2016b) sostienen que valores mayores a .70 indican que la variable latente se encuentra bien definida por sus indicadores $y$, por lo tanto, existe apoyo a su replicabilidad. En relación con lo anterior, también resulta importante determinar si la escala es predominantemente unidimensional y, en consecuencia, no es necesario modelar todos los factores específicos en un modelo SEM. Para tomar esta decisión, es posible examinar de manera conjunta dos índices: la varianza común explicada $(E C V)$ y el porcentaje de correlaciones no contaminadas (PUC). La ECV estima el porcentaje de la varianza común que es explicado por el factor general y, por lo tanto, indica la medida en que las cargas factoriales se mantienen insesgadas cuando el modelo bifactor es forzado en un modelo unidimensional. Por su parte, el $P U C$ señala qué proporción de las correlaciones interítem ocurre entre ítems de factores específicos distintos; dado que estos no comparten otra variable latente aparte del factor general, un valor elevado de $P U C$ señala la preponderancia de este. El trabajo de Rodriguez et al. (2016a) sugiere que valores $E C V$ y $P U C$ mayores a .70 indican que es posible forzar el modelo bifactor en uno unidimensional sin que esto implique un gran sesgo.
A continuación, se procedió a ejecutar un análisis exploratorio bifactor. La razón de realizar esto es que, cuando se realiza un análisis restringido (confirmatorio), es posible que existan especificaciones erróneas que no se evidencian en los índices de ajuste (Reise, 2012). El análisis se basó en correlaciones policóricas y usó el estimador WLSMV. Se utilizó una rotación procusteana semiespecificada ortogonal (Browne, 1972), la cual partió de una matriz diana basada en la estructura original de la prueba (Lovibond \& Lovibond, 1995). El objetivo de esta rotación es reducir la distancia entre la solución factorial rotada y la matriz diana (Ferrando \& Lorenzo-Seva, 2014). Con fines exploratorios, se permitió la modificación de la matriz diana a través del método RETAM (LorenzoSeva \& Ferrando, 2019).

Se utilizó los paquetes psych (Revelle, 2018) y lavaan (Rosseel, 2012) -implementados en el programa estadístico $\mathrm{R}$, versión 3.5.3 ( $\mathrm{R}$ Core Team, 2019) - para los análisis descriptivos y el AFC. Además, se utilizó el módulo elaborado por Domínguez-Lara y Rodriguez (2017) para calcular los índices correspondientes al modelo bifactor. El análisis exploratorio bifactor se llevó a cabo en el programa FACTOR (Lorenzo-Seva \& Ferrando, 2013), versión 10.9.02.

\section{RESULTADOS}

Se analizó los estadísticos descriptivos de los ítems del instrumento. En todos los casos, las medias y las desviaciones estándar fueron inferiores a 1. Asimismo, al examinar los porcentajes de respuesta que recibió cada una de las cuatro opciones, se observó que en la mayoría de los casos - la mayor proporción de respuestas correspondió a la primera opción (No describe nada de lo que me pasó o sentí en la semana). Solo cinco ítems $(5,6,8,11$ y 12) obtuvieron la mayor cantidad de respuestas en la segunda opción (Sí, esto me pasó o lo sentí en alguna medida o en algún momento). De estos cinco ítems, cuatro provinieron de la escala Estrés. Ningún ítem mostró altos porcentajes de respuesta en la tercera o en la cuarta opción.

Dada la naturaleza ordinal de los indicadores, se procedió a realizar un AFC con el estimador WLSMV. En la Tabla 2, se presentan los índices 
de ajuste obtenidos por cada uno de los modelos probados. El modelo unidimensional (M1) obtuvo un ajuste subóptimo. Cuando se especificó un modelo de dos factores - activación fisiológica y afectividad general (M2) -, se apreció un ajuste mejor pero aún inadecuado, con una correlación interfactorial elevada $(\Phi=.794)$. Otro modelo de dos factores que se puso a prueba fue el de un factor de depresión y otro de ansiedad-estrés (M3), el cual obtuvo un mejor ajuste que los anteriores, aunque se evidenció una correlación interfactorial también elevada $(\Phi=.799)$. El siguiente modelo probado fue el propuesto originalmente por los autores de la prueba (M4) y consta de tres factores - depresión, ansiedad y estrés - que se encuentran correlacionados. Este modelo evidenció un mejor ajuste pero aún se encontraron altas correlaciones interfactoriales $(\Phi \mathrm{D}, \mathrm{A}=.855, \Phi \mathrm{D}, \mathrm{E}=.718, \Phi \mathrm{A}, \mathrm{E}$ $=.905)$. A partir de lo expuesto, se probó un modelo bifactor (M5), el cual obtuvo un ajuste superior al de todos los modelos probados.

Tabla 2

Índices de ajuste de los modelos probados

\begin{tabular}{lccccccc}
\hline Modelo & WLSMV $\chi^{2}$ & $\mathrm{gl}$ & CFI & TLI & RMSEA & SRMR & WRMR \\
\hline M1 & 607.00 & 189 & .916 & .906 & .079 & .077 & 1.37 \\
M2 & 547.28 & 188 & .927 & .919 & .074 & .072 & 1.29 \\
M3 & 459.62 & 188 & .945 & .939 & .064 & .064 & 1.13 \\
M4 & 396.80 & 186 & .957 & .952 & .057 & .058 & 1.02 \\
M5 & 319.56 & 168 & .969 & .962 & .051 & .048 & 0.85 \\
\hline
\end{tabular}

La primera parte de la Tabla 3 ("Confirmatorio") contiene las cargas factoriales estimadas en los cuatro factores del modelo bifactor (uno general y tres específicos). Con base en estas cargas factoriales estandarizadas, se calcularon los índices correspondientes $(\omega, \omega \mathrm{H}$ y $\mathrm{H})$. Se observa que, en conjunto, los factores del modelo explicaron la mayor parte de la varianza de los ítems $(\omega>.90)$. Sin embargo, cuando se analizó el aporte de cada factor por separado $(\omega \mathrm{H})$, controlando la influencia de los otros factores, solo el factor general de malestar emocional explicó una proporción significativa de la varianza.
Un patrón similar se halló al examinar la replicabilidad del constructo $(\mathrm{H})$ y el promedio de cargas factoriales. En todos los casos, el factor específico que evidenció mayores valores - aunque menores que los del factor general- fue el de depresión (Tabla 4). Por otra parte, los valores ECV $=.764$ y $\mathrm{PUC}=.700$ indicaron que podía forzarse un modelo unidimensional sin que esto implicara una pérdida importante de información.

A continuación, se realizó un análisis factorial exploratorio bifactor, para el cual se estableció una matriz diana consistente en un factor general y los tres factores específicos de depresión, ansiedad y estrés. Antes de la rotación, la matriz diana se refinó con el método RETAM, permitiendo la estimación libre del ítem 15 en el factor Estrés, además de su factor original (Ansiedad). Los resultados indicaron que todos los ítems alcanzaron cargas notablemente mayores en el factor general (Tabla 3, "Exploratorio"). Sin embargo, al examinar las cargas factoriales de los factores específicos, se observó que los ítems 9, 15 y 20 tuvieron su mayor carga positiva en factores distintos al hipotetizado. 
Tabla 3

Análisis bifactor de las escalas DASS-21

\begin{tabular}{|c|c|c|c|c|c|c|c|c|}
\hline \multirow[t]{2}{*}{ Ítem } & \multicolumn{4}{|c|}{ Confirmatorio } & & \multicolumn{3}{|c|}{ Exploratorio } \\
\hline & FG & $\mathrm{D}$ & A & $\mathrm{E}$ & FG & $\mathrm{D}$ & $\mathrm{A}$ & $\mathrm{E}$ \\
\hline 1. Estrés & .592 & & & .408 & .621 & -.100 & .091 & .379 \\
\hline 2. Ansiedad & .535 & & .257 & & .426 & .087 & .357 & .154 \\
\hline 3. Depresión & .658 & .213 & & & .540 & .311 & .247 & .159 \\
\hline 4. Ansiedad & .613 & & .478 & & .518 & .040 & .588 & .101 \\
\hline 5. Depresión & .567 & .274 & & & .610 & .226 & .009 & -.014 \\
\hline 6. Estrés & .564 & & & .394 & .477 & .116 & .047 & .489 \\
\hline 7. Ansiedad & .602 & & .389 & & .583 & .034 & .443 & -.040 \\
\hline 8. Estrés & .714 & & & .290 & .759 & -.146 & .165 & .280 \\
\hline 9. Ansiedad & .749 & & -.020 & & .714 & -.024 & .120 & .220 \\
\hline 10. Depresión & .640 & .502 & & & .763 & .401 & -.096 & -.094 \\
\hline 11. Estrés & .746 & & & .294 & .751 & -.032 & .102 & .359 \\
\hline 12. Estrés & .684 & & & .468 & .607 & .036 & .080 & .590 \\
\hline 13. Depresión & .652 & .322 & & & .507 & .467 & .035 & .317 \\
\hline 14. Estrés & .691 & & & .158 & .463 & .259 & .243 & .481 \\
\hline 15. Ansiedad & .845 & & -.148 & & .598 & .301 & .212 & .408 \\
\hline 16. Depresión & .540 & .343 & & & .412 & .467 & .221 & .119 \\
\hline 17. Depresión & .671 & .562 & & & .642 & .603 & .131 & .099 \\
\hline 18. Estrés & .607 & & & .381 & .478 & .131 & .144 & .527 \\
\hline 19. Ansiedad & .730 & & .475 & & .666 & .012 & .580 & .053 \\
\hline 20. Ansiedad & .821 & & .028 & & .663 & .312 & .235 & .190 \\
\hline 21. Depresión & .675 & .637 & & & .677 & .651 & .214 & -.021 \\
\hline$\omega$ & .960 & .905 & .903 & .897 & & & & \\
\hline$\omega \mathrm{H}$ & .886 & .268 & .074 & .191 & & & & \\
\hline $\mathrm{H}$ & .950 & .650 & .462 & .511 & & & & \\
\hline
\end{tabular}

Nota.FG: Factor general, D: Depresión, A: Ansiedad, E: Estrés. Se resalta en negrita la mayor carga factorial positiva en un factor específico.

\section{DISCUSIÓN}

El presente estudio tuvo por fin analizar la dimensionalidad de las escalas DASS-21. Se realizó, en primer lugar, un AFC, el cual indicó que el modelo de tres factores específicos y uno general obtenía el mejor ajuste. Sin embargo, solo el factor general denominado malestar emocional - explicaba la mayor parte de la varianza de los indicadores. Un patrón similar se halló al realizar un análisis factorial exploratorio, aunque no todos los ítems correspondieron a sus factores específicos originales.
La bibliografía sugiere que existe un notable solapamiento entre los factores de la DASS-21 (Moussa et al., 2017; Page et al., 2007; Patias et al., 2016; Sinclair et al., 2012; Szabó, 2010; Wang et al., 2016). En el presente estudio, todos los modelos que incluyeron más de un factor evidenciaron correlaciones interfactoriales elevadas. En el modelo de tres factores (Lovibond \& Lovibond, 1995), la mayor correlación se halló entre los factores de ansiedad y estrés, lo que indicaría la posibilidad de fusionar estos en uno solo. Cuando esta fusión se realizó (M3), la correlación 
entre el factor ansiedad-estrés y el de depresión fue también elevada.

Las altas correlaciones interfactoriales llevan a considerar la posibilidad de un factor general en conjunto con los tres factores específicos de ansiedad, depresión y estrés (Alfonsson et al., 2017; Bottesi et al., 2015; Henry \& Crawford, 2005; Kia-Keating et al., 2018; Moore et al., 2017; Osman et al., 2012; Shaw et al., 2016; Szabó, 2010; Tully et al., 2009). En el presente estudio, esto se puso a prueba con un modelo bifactor (M5). El factor general -denominado aquí malestar emocional - ha sido equiparado, en ocasiones, al afecto negativo del modelo tripartito de la ansiedad y la depresión (Bottesi et al., 2015). Según este modelo, la ansiedad y la depresión comparten una dimensión general (el afecto negativo), pero poseen, también, un aspecto específico a cada una de ellas: la hiperactivación fisiológica en el caso de la ansiedad y el bajo afecto positivo en el caso de la depresión (Clark \& Watson, 1991). En conceptualizaciones tempranas de la escala DASS, se propuso una equivalencia entre la escala Estrés y el afecto negativo (Brown et al., 1997). Sin embargo, esta equivalencia no logró ser replicada (Norton, 2007) y, cuando se aplicaron los modelos bifactor, se demostró que el estrés constituye una dimensión específica independiente del afecto negativo (Bottesi et al., 2015; Szabó, 2010). En los presentes datos, esto se refleja en los valores de $\omega \mathrm{H}$ y $\mathrm{H}$ del factor específico de estrés, los cuales aunque fueron bajos en comparación con los del factor general-, mostraron valores superiores a cero $\mathrm{y}$ mayores que los que obtuvo la escala ansiedad.

Llaman la atención los bajos valores de los coeficientes pertenecientes al factor específico de ansiedad. De acuerdo con el modelo tripartito, este correspondería principalmente al componente de hiperactivación fisiológica. Al analizar las cargas factoriales de los ítems de este factor específico, se observó que tres de ellos $(9,15,20)$ obtuvieron cargas factoriales muy bajas o negativas, lo cual indica que constituyen indicadores puros del factor general (Kia-Keating et al., 2018; Moore et al., 2017; Shaw et al., 2016). Estos mismos ítems (especialmente, el 15) tuvieron su mayor carga en un factor distinto al hipotetizado en el análisis exploratorio. Por otra parte, los ítems restantes (2, 4, 7 y 19) alcanzaron cargas factoriales mayores. Al analizar su contenido, se observó que los cuatro ítems describían claramente experiencias de alteración fisiológica en consonancia con el modelo tripartito. De hecho, algunos autores han identificado estos mismos ítems como los más representativos de la escala ansiedad (Osman et al., 2012; Shaw et al., 2016). Sin embargo, se debe considerar el carácter problemático del ítem 2 , el cual ha mostrado bajas cargas factoriales y pobre capacidad discriminativa en algunos estudios (Clara et al., 2001; Kia-Keating et al., 2018; Patias et al., 2016; Sinclair et al., 2012).

En relación a la escala Depresión, cabe mencionar que investigaciones previas han hecho notar la mayor capacidad discriminativa de esta respecto de las otras dos escalas (Moore et al., 2017; Sinclair et al., 2012; Szabó, 2010; Wang et al., 2016). En los presentes resultados, esto se evidencia en los mayores valores de $\omega \mathrm{H}$ y $\mathrm{H}$ que presentó el factor específico correspondiente a esta escala. De acuerdo con el modelo tripartito, el componente específico de la depresión es la anhedonia o bajo afecto negativo (Clark \& Watson, 1991). Por lo tanto, se esperaría que los ítems con mayores cargas factoriales en el factor específico de depresión mostrasen en su contenido descripciones de la anhedonia. Estos ítems (10, 16, $17,21)$ coincidieron en su mayoría con lo hallado en estudios previos (Osman et al., 2012; Shaw et al., 2016) y mostraron, en efecto, una correspondencia parcial con el constructo de anhedonia, pero, también, por ejemplo, con el de desesperanza.

En relación a la escala Estrés, se observó que las mayores cargas factoriales en el factor específico correspondieron a los ítems $1,6,12$ y 18 , lo cual coincide en gran medida con lo hallado en otros estudios (Osman et al., 2012). Asimismo, el ítem 1 parece ser el más representativo de dicho factor específico (KiaKeating et al.,2018; Shaw et al., 2016). Estos resultados indican que, aunque la mayor parte de la varianza es explicada por el factor general, existe un porcentaje residual que corresponde al factor específico de estrés, el cual, por lo tanto, no es completamente reducible a una dimensión global de afecto negativo (Bottesi et al., 2015; Szabó, 2010). A pesar de ello, según se observa en los análisis del modelo bifactor, en términos prácticos, el factor general de malestar emocional es 
el único que justifica la formación de una puntuación compuesta y el modelamiento de una variable latente en un modelo estructural. Es posible, en el futuro, poner a prueba modelos ultrabreves conformados por los ítems más representativos de cada factor específico y observar si, en estas situaciones, la preponderancia del factor general disminuye (Kia-Keating et al., 2018; Kyriazos et al, 2018; Lee et al., 2019; Moore et al., 2017).

La presente investigación posee algunas limitaciones. Un hecho notorio es que la muestra con la que se trabajó estuvo constituida en su totalidad por estudiantes de Psicología, por lo cual los resultados no pueden ser extrapolados ni a estudiantes de otras carreras ni a la población general. Asimismo, el tamaño de la muestra no permitió realizar un análisis de invarianza entre varones y mujeres ( $\mathrm{Lu}$ et al., 2018) ni permitió que los análisis confirmatorio y exploratorio se realizaran en submuestras distintas. Además, si bien el estimador utilizado para realizar el AFC (WLSMV) es considerado el más adecuado cuando se trabaja con indicadores de naturaleza ordinal (Brown, 2015; Byrne, 2012), existen al menos tres limitaciones analíticas asociadas a este estimador. En primer lugar, por definición, el estimador WLSMV no analiza las variables observadas ordinales, sino las hipotéticas variables continuas que subyacen a estas (Byrne, 2012). Los coeficientes $\omega$ y $\omega \mathrm{H}$ calculados en el presente estudio, por lo tanto, no indicarían la confiabilidad de las sumas de puntuaciones observadas - como es su finalidad - sino la de hipotéticas puntuaciones compuestas de las variables subyacentes. La segunda limitación del uso de WLSMV es que, aunque no se requiere que los puntajes observados se ajusten a la normalidad, sí se asume que la hipotética variable continua que subyace a estas puntuaciones presenta normalidad multivariada. Sin embargo, la descripción de síntomas psicopatológicos de ansiedad y depresión en una población no clínica de estudiantes hace que este supuesto sea difícil de asumir (Rhemtulla, BrosseauLiard, \& Savalei, 2012). Finalmente, es importante tener en cuenta que los puntos de corte utilizados para evaluar los índices de ajuste (p. ej., CFI, RMSEA) fueron obtenidos a partir de simulaciones con el estimador de máxima verosimilitud aplicado a un conjunto específico de modelos (Hu \& Bentler, 1999). Por tal motivo, la aplicación de estos puntos de corte a condiciones distintas - como es el análisis de modelos bifactor con el estimador WLSMV - debe tomarse con cautela (Greiff \& Heene, 2017). A pesar de estas limitaciones, la literatura actualizada considera que, a la fecha, WLSMV constituye la mejor alternativa para realizar análisis factoriales de variables ordinales (Brown, 2015) y, en consecuencia, gran parte de los estudios recientes sobre la escala DASS-21 ha utilizado este estimador (Bottesi et al., 2015; Kia-Keating et al., 2018; Mellor et al., 2015; Moore et al., 2017; Shaw et al., 2016).

En conclusión, los resultados del presente estudio indican que la estructura de la escala DASS21 es, principalmente, unidimensional, aunque con la presencia de algunos factores específicos de naturaleza residual. De estos factores específicos, es el factor depresión el que requiere mayor análisis como una posible dimensión independiente. Estudios futuros deben examinar la posibilidad de generar versiones ultrabreves que delimiten mejor la estructura de los factores específicos de la DASS-21. Por lo expuesto, se sugiere que, cuando se utilicen la escala DASS21 en muestras similares a las de este estudio, se calcule un puntaje general en lugar de los tres puntajes específicos originales.

\section{REFERENCIAS}

Alfonsson, S., Wallin, E., \& Maathz, P. (2017). Factor structure and validity of the Depression, Anxiety and Stress Scale-21 in Swedish translation.Journal of Psychiatric and Mental Health Nursing, 24(23), 154-162. doi: 10.1111/jpm.12363

Antony, M. M., Bieling, P. J., Cox, B. J., Enns, M. W., \& Swinson, R. P. (1998). Psychometric properties of the 42-item and 21-item versions of the Depression Anxiety Stress Scales in clinical groups and a community sample. Psychological Assessment, 10(2), 176-181.

Asociación Americana de Psiquiatría (2014). Manual diagnóstico yestadístico de los trastornosmentales

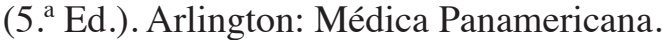

Bados, A., Solanas, A., \& Andrés, R. (2005). Psychometric properties of the Spanish version of Depression, Anxiety and Stress Scales (DASS). Psicothema, 17(4), 679-683. 
Barlow, D. H., \& Durand, V. M. (2015). Abnormal psychology: an integrated approach (7. ${ }^{\mathrm{a}} \mathrm{Ed}$.). Stamford: Cengage Learning.

Bottesi, G., Ghisi, M., Altoè, G., Conforti, E., Melli, G., \& Sica, C. (2015). The Italian version of the Depression Anxiety Stress Scales-21: Factor structure and psychometric properties on community and clinical samples. Comprehensive Psychiatry, 60, 170-181. doi: 10.1016/j. comppsych.2015.04.005

Brown, T. A. (2015). Confirmatory factor analysis for applied research (2. ${ }^{\mathrm{a}}$ Ed.). Nueva York: The Guilford Press.

Brown, T. A., Chorpita, B. F., Korotitsch, W., \& Barlow, D. H. (1997). Psychometric properties of the Depression Anxiety Stress Scales (DASS) in clinical samples. Behaviour Research and Therapy, 35(1), 79-89. doi: 10.1016/S00057967(96)00068-X

Browne,M.W.(1972). Orthogonal rotation to a partially specified target. British Journal of Mathematical and Statistical Psychology, 25(1), 115-120. doi: 10.1111/j.2044-8317.1972.tb00482.x

Byrne, B. M. (2012). Structural equation modeling with Mplus: Basic concepts, applications, and programming. Nueva York: Routledge.

Camacho, Á., Cordero, E. D., \& Perkins, T. (2016). Psychometric properties of the DASS-21 among Latina/o college students by the US-Mexico border. Journal of Immigrant and Minority Health, 18(5), 1017-1023. doi:10.1007/s10903016-0415-1

Clara, I. P., Cox, B. J., \& Enns, M. W. (2001). Confirmatory factor analysis of the DepressionAnxiety-Stress Scales in depressed and anxiety patients. Journal of Psychopathology and Behavioral Assessment, 23(1), 61-67. doi: 10.1023/A:1011095624717

Clark, L. A., \& Watson, D. (1991). Tripartite model of anxiety and depression: Psychometric evidence and taxonomic implications. Journal of Abnormal Psychology, 100(3), 316-336. doi: 10.1037/0021843X.100.3.316
Daza, P., Novy, D.M., Stanley, M.A., \& Averill, P. (2002). The Depression Anxiety Stress Scale-21: Spanish translation and validation with a Hispanic sample. Journal of Psychopathology and Behavioral Assessment, 24(3), 195-205. doi: /10.1023/A:1016014818163

DiStefano, C., Liu, J., Jiang, N., \& Shi, D. (2018). Examination of the Weighted Root Mean Square Residual: Evidence for trustworthiness? Structural Equation Modeling: A Multidisciplinary Journal, 25(3), 453-466. doi: 10.1080/10705511.2017.1390394

Dominguez-Lara, S. A. (2016). Evaluación de la confiabilidad del constructo mediante el coeficiente $\mathrm{H}$ : Breve revisión conceptual y aplicaciones. Psychologia. Avances en la Disciplina, 10(2), 8794. doi: 10.21500/19002386.2134

Dominguez-Lara, S. A., \& Rodriguez, A. (2017). Índices estadísticos de modelos bifactor. Interacciones, 3(2), 59-65. doi: 10.24016/2017. v3n2.51

Duffy, C. J., Cunningham, E. G., \& Moore, S. M. (2005). Brief report: The factor structure of mood states in an early adolescent sample. Journal of Adolescence, 28(5), 677-680. doi: 10.1016/j. adolescence. 2005.08 .013

Eysenck, M. W., \& Fajkowska, M. (2018). Anxiety and depression: toward overlapping and distinctive features. Cognition and Emotion, 32(7), 13911400. doi: 10.1080/02699931.2017.1330255

Ferrando, P. J., \& Lorenzo-Seva, U. (2014). El Análisis Factorial Exploratorio de los Ítems: algunas consideraciones adicionales. Anales de Psicología, 30(3), 1170-1175. doi: 10.6018/ analesps.30.3.199991

Greiff, S., \& Heene, M. (2017). Why psychological assessment needs to start worrying about model fit. European Journal of Psychological Assessment, 33(5), 313-317. doi: 10.1027/1015-5759/a000450

Gurrola, G. M., Balcázar, P., Bonilla, M. P., \& Virseda, J. A. (2006). Estructura factorial y consistencia interna de la Escala de Depresión Ansiedad y Estrés (DASS-21) en una muestra no clínica. Psicología y Ciencia Social, 8(2), 3-7. 
Hancock, G. R., \& Mueller, R. O. (2001). Rethinking construct reliability within latent variable systems. En R. Cudeck, S. du Toit, \& D. Sörbom (Eds.), Structural equation modeling: Present and future-A festschrift in honor of Karl Jöreskog (pp. 195-216). Lincolnwood: Scientific Software International.

Henry, J. D., \& Crawford, J. R. (2005). The short-form version of the Depression Anxiety Stress Scales (DASS-21): Construct validity and normative data in a large non-clinical sample. British Journal of Clinical Psychology, 44(2), 227-239. doi: 10.1348/014466505X29657

Hu,L.,\& Bentler,P.(1999).Cutoffcriteria for fit indexes in covariance structure analysis: Conventional criteria versus new alternatives. Structural Equation Modeling: A Multidisciplinary Journal, 6(1), 1-55. doi: 10.1080/10705519909540118

Jun, D., Johnston, V., Kim, J. M., \& O'Leary, S. (2018). Cross-cultural adaptation and validation of the Depression, Anxiety and Stress Scale-21 (DASS-21) in the Korean working population. Work, 59(1), 93-102. doi: 10.3233/WOR-172661

Kia-Keating, M., No, U., Moore, S., Furlong, M., Liu, S., \& You, S. (2018). Structural validity of the Depression, Anxiety, and Stress Scales-21 adapted for U.S. undergraduates. Emerging Adulthood, 6(6), 434-440. doi: 10.1177/2167696817745407

Kyriazos, T. A., Stalikas, A., Prassa, K., \& Yotsidi, V. (2018). Can the Depression Anxiety Stress Scales short be shorter? Factor structure and measurement invariance of DASS-21 and DASS-9 in a Greek, non-clinical sample. Psychology, 9(5), 10951127. https://doi.org/10.4236/psych.2018.95069

Le, M. T. H., Tran, T. D., Holton, S., Nguyen, H. T., Wolfe, R., \& Fisher, J. (2017). Reliability, convergent validity and factor structure of the DASS-21 in a sample of Vietnamese adolescents. PLoS One, 12(7), e0180557. doi: 10.1371/journal. pone. 0180557

Lee, E. H., Moon, S. H., Cho, M. S., Park, E. S., Kim, S. Y., Han, J. S., \& Cheio, J. H. (2019). The 21item and 12-item versions of the Depression Anxiety Stress Scales: Psychometric evaluation in a Korean population. Asian Nursing Research, 13(1), 30-37. doi: 10.1016/j.anr.2018.11.006
Lorenzo-Seva, U., \& Ferrando, P. J. (2013). FACTOR 9.2: A comprehensive program for fitting exploratory and semiconfirmatory factor analysis and IRT models. Applied Psychological Measurement, 37(6), 497-798. doi: /10.1177/0146621613487794

Lorenzo-Seva, U., \& Ferrando, P. J. (2019). Unrestricted factor analysis of multidimensional test items based on an objectively refined target matrix. Behavior Research Methods. Publicación anticipada en línea. doi: 10.3758/s13428-01901209-1

Lovibond,P.F., \& Lovibond, S.H.(1995). The structure of negative emotional states: Comparison of the Depression Anxiety Stress Scales (DASS) with the Beck Depression and Anxiety Inventories. Behaviour Research and Therapy, 33(3), 335343. doi: 10.1016/0005-7967(94)00075-U

Lu, S., Hu, S., Guan, Y., Xiao, J., Cai, D., Gao, Z., Margraf, J. (2018). Measurement invariance of the Depression Anxiety Stress Scales-21 across gender in a sample of Chinese university students. Frontiers in Psychology, 9, 2064. doi: 10.3389/ fpsyg.2018.02064

Mellor, D., Vinet, E. V., Xu, X., Bt Mamat, N. H., Richardson, B., \& Román, F. (2015). Factorial invariance of the DASS-21 among adolescents in four countries. European Journal of Psychological Assessment, 31(2), 138-142. doi: 10.1027/10155759/a000218

Moore, S.A.,Dowdy, E., \& Furlong, M. J.(2017).Using the Depression, Anxiety, Stress Scales-21 with U.S. adolescents: An alternate models analysis. Journal of Psychoeducational Assessment, 35(6), 581-598. doi: 10.1177/0734282916651537

Moussa, M. T., Lovibond, P., Laube, R., \& Megahead, H.A. (2017). Psychometric properties of an Arabic version of the Depression Anxiety Stress Scales (DASS). Research on Social Work Practice, 27(3), 375-386. doi: 10.1177/1049731516662916

Norton, P. J. (2007). Depression Anxiety and Stress Scales (DASS-21): Psychometric analysis across four racial groups. Anxiety, Stress, \& Coping, 20(3), 253-265 doi: 10.1080/10615800701309279 
Osman, A., Wong, J. L., Bagge, C. L., Freedenthal, S., Gutierrez, P. M., \& Lozano, G. (2012). The Depression Anxiety Stress Scales-21 (DASS21): Further examination of dimensions, scale reliability, and correlates. Journal of Clinical Psychology, 68(12), 1322-1338. doi: 10.1002/ jclp.21908

Page, A. C., Hooke, G. R., \& Morrison, D. L. (2007). Psychometric properties of the Depression Anxiety Stress Scales (DASS) in depressed clinical samples. British Journal of Clinical Psychology, 46(3), 283-297. doi:10.1348/014466506X158996

Patias, N. D., Machado, W. L., Bandeira, D. R., \& Dell'Aglio, D. D. (2016). Depression Anxiety and Stress Scale (DASS-21) - Short Form: Adaptação e validação para adolescentes brasileiros. Psico-USF, 21(3), 459-469. doi: 10.1590/1413-82712016210302

Patrick, J., Dyck, M., \& Bramston, P. (2010). Depression Anxiety Stress Scale: Is it valid for children and adolescents? Journal of Clinical Psychology, 66(9), 996-1007. doi: 10.1002/ jclp.20696

Randall, D., Thomas, M., Whiting, D., \& McGrath, A. (2017). Depression Anxiety Stress Scales (DASS-21): Factor structure in traumatic brain injury rehabilitation. The Journal of Head Trauma Rehabilitation, 32(2), 134-144. doi: 10.1097/ HTR.0000000000000250

R Core Team (2019). R: A language and environment for statistical computing (versión 3.5.3) [Programa de computadora]. Viena: R Foundation for Statistical Computing.

Reise, S. P. (2012). The rediscovery of bifactor measurement models. Multivariate Behavioral Research, 47(5), 667-696. doi: 10.1080/00273171.2012.715555

Reise, S. P., Bonifay, W. R., \& Haviland, M. G. (2013). Scoring and modeling psychological measures in the presence of multidimensionality. Journal of Personality Assessment, 95(2), 129-140. doi: 10.1080/00223891.2012.725437

Rhemtulla, M., Brosseau-Liard, P. E. \& Savalei, V. (2012). When can categorical variables be treated as continuous? A comparison of robust continuous and categorical SEM estimation methods under suboptimal conditions. Psychological Methods, 17(3), 354-373. doi: 10.1037/a0029315

Revelle, W. (2018). psych: Procedures for Personality and Psychological Research. Paquete de R, versión 1.8.4. https://CRAN.R-project.org/ package $=$ psych

Rodriguez, A., Reise, S. P., \& Haviland, M. G. (2016a). Applying bifactor statistical indices in the evaluation of psychological measures. Journal of Personality Assessment, 98(3), 223-237. doi: 10.1080/00223891.2015.1089249

Rodriguez, A., Reise, A. P., \& Haviland, M. G. (2016b). Evaluating bifactor models: Calculating and interpreting statistical indices. Psychological Methods, 21(2), 137-150. doi: 10.1037/ met0000045

Román, F., Vinet, E. V., \& Alarcón, A. M. (2014). Escalas de Depresión, Ansiedad y Estrés (DASS21): Adaptación y propiedades psicométricas en estudiantes secundarios de Temuco. Revista Argentina de Clínica Psicológica, 23(2), 179-190.

Rosseel, Y. (2012). lavaan: An R Package for Structural Equation Modeling. Journal of Statistical Software, 48(2), 1-36. doi: 10.18637/jss.v048.i02

Ruiz, F. J., García Martín, M. B., Suárez Falcón, J. C., \& Odriozola González, P. (2017). The hierarchical factor structure of the Spanish version of Depression Anxiety and Stress Scale -21. International Journal of Psychology and Psychological Therapy, 17(1), 97-105.

Sandín, B., \& Chorot, P. (2009). Concepto y categorización de los trastornos de ansiedad. En A. Belloch, B. Sandín y F. Ramos (Coords.), Manual de psicopatología. Vol. 2 (pp. 43-63). Madrid: McGraw-Hill/Interamericana.

Scholten, S., Velten, J., Bieda, A., Zhang, X. C., \& Margraf, J. (2017). Testing measurement invariance of the Depression, Anxiety, and Stress Scales (DASS-21) across four countries. Psychological Assessment, 29(11), 1376-1390. doi: $10.1037 /$ pas0000440

Shaw, T., Campbell, M. A., Runions, K. C., \& Zubrick, S. R. (2016). Properties of the DASS-21 in an 
Australian community adolescent population. Journal of Clinical Psychology, 73(7), 879-892. doi: 10.1002/jclp.22376

Sinclair, S. J., Siefert, C. J., Slavin-Mulford, J. M., Stein, M. B., Renna, M., \& Blais, M. A. (2012). Psychometric evaluation and normative data for the Depression, Anxiety, and Stress Scales-21 (DASS-21) in a nonclinical sample of U.S. adults. Evaluation \& the Health Professions, 35(3), 259279. doi: 10.1177/0163278711424282

Szabó, M. (2010). The short version of the Depression Anxiety Stress Scales (DASS-21): Factor structure in a young adolescent sample. Journal of Adolescence, 33(1), 1-8. doi: 10.1016/j. adolescence.2009.05.014

Szabó, M., \& Lovibond, P. F. (2006). Anxiety, depression and tension/stress in children. Journal of Psychopathology and Behavioral Assessment, 28(3), 195-205. doi: 10.1007/s10862-005-9008-3

Tran, T. D., Tran, T., \& Fisher, J. (2013). Validation of the depression anxiety stress scales (DASS) 21 as a screening instrument for depression and anxiety in a rural community-based cohort of northern Vietnamese women. BMC Psychiatry, 13, artículo 24. doi: 10.1186/1471-244X-13-24

Tully, P. J., Zajac, I. A., \& Venning, A. J. (2009). The structure of anxiety and depression in a normative sample of younger and older Australian adolescentes. Journal of Abnormal Child Psychology, 37(5), 717-726. doi: 10.1007/ s10802-009-9306-4
Vásquez, C., \& Sanz, J. (2009). Trastornos del estado de ánimo: Aspectos clínicos. En A. Belloch, B. Sandín y F. Ramos (Coords.), Manual de psicopatología. Vol. 2 (pp. 233-269). Madrid: McGraw-Hill/Interamericana.

Wang, K., Shi, H. S., Geng, F. L., Zou, L. Q., Tan, S. P., Wang, Y., ... Chan, R. C. K. (2016). Cross-cultural validation of the Depression Anxiety Stress Scale-21 in China. Psychological Assessment, 28(5), e88-e100. doi: 10.1037/pas0000207

Watson, D., \& Clark, L.A. (1984). Negative affectivity: the disposition to experience aversive emotional states. Psychological Bulletin, 96(3), 465-490. I doi: 10.1037/0033-2909.96.3.465

Willemsen, J., Markey, S., Declercq, F., \& Vanheule, S. (2011). Negative emotionality in a large community sample of adolescents: the factor structure and measurement invariance of the short version of the Depression Anxiety Stress Scales (DASS-21). Stress and Health, 27(3), e120-e128. doi: $10.1002 /$ smi.1342

Yıldırım, A., Boysan, M., \& Kefeli, M. C. (2018). Psychometric properties of the Turkish version of the Depression Anxiety Stress Scale-21 (DASS-21). British Journal of Guidance \& Counselling, 46(5), 582-595. doi: 10.1080/03069885.2018.1442558

Fecha de recepción: 29 de mayo 2019

Fecha de aceptación: 27 de junio 2019 